Slavica

bruxellensia

\section{Slavica bruxellensia}

Revue polyphonique de littérature, culture et histoire

slaves

$10 \mid 2014$

Espace slave, espace germanique

\title{
Les emprunts allemands dans la presse russe actuelle
}

\section{Valentina Caillat-Tuzhikova}

\section{OpenEdition}

\section{Journals}

Édition électronique

URL : http://journals.openedition.org/slavica/1634

DOI : $10.4000 /$ slavica. 1634

ISSN : 2034-6395

\section{Éditeur}

Université libre de Bruxelles - ULB

\section{Référence électronique}

Valentina Caillat-Tuzhikova, « Les emprunts allemands dans la presse russe actuelle», Slavica bruxellensia [En ligne], 10 | 2014, mis en ligne le 15 avril 2014, consulté le 19 avril 2019. URL : http:// journals.openedition.org/slavica/1634; DOI : 10.4000/slavica.1634

Ce document a été généré automatiquement le 19 avril 2019

\section{(c) $(1) \odot$}

Les contenus de Slavica bruxellensia sont mis à disposition selon les termes de la Licence Creative Commons Attribution - Pas d'Utilisation Commerciale - Pas de Modification 3.0 France. 


\title{
Les emprunts allemands dans la presse russe actuelle
}

\author{
Valentina Caillat-Tuzhikova
}

\section{Introduction}

1 À l'heure actuelle, la presse russe représente un champ d'intérêt incontournable pour l'étude des emprunts. En raison des changements politiques et sociaux que la Russie a subis durant ces deux dernières décennies, il est devenu nécessaire de nommer des objets ou des phénomènes nouveaux. La langue russe a ainsi accéléré le processus d'appropriation de mots étrangers pour combler des lacunes dans le vocabulaire politique, économique et juridique. La presse russe s'est dès lors approprié ces nouveaux mots et en a introduit d'autres à son tour. Parmi les langues étrangères concernées, l'allemand est une des sources majeures de ce vocabulaire contemporain. Quels sont les emprunts allemands et quels sens possèdent-ils dans la langue source? Comment ces mots évoluent-ils en russe ? Y-a-il un lien de dépendance entre la fréquence d'utilisation des emprunts et leur appropriation par la langue ? Les questions que nous analyserons ici concernent l'emploi des emprunts allemands dans la presse et leur évolution sur le plan sémantique et en particulier connotatif.

Un bref rappel historique permet de comprendre l'important impact des emprunts allemands dans la langue russe.Le XIII siècle est considéré, par l'ensemble des linguistes, comme le point de départ de l'assimilation des mots allemands dans la langue russe, et ce, en raison des rapports commerciaux étroits entre l'Allemagne et la Rus' de Kiev. Ensuite, l'époque de Pierre le Grand marque l'introduction massive du lexique allemand dans le russe. Comme le précise Irina Tokareva dans sa thèse Adaptacija nemeckikh leksičeskikh zaimstvovanij $v$ russkom literaturnom jazyke: Na materiale istočnikov rubeža XIX - XX vv. (L'adaptation des emprunts lexicaux dans la langue russe littéraire : Sources des XIXe et $\mathrm{XX}^{\mathrm{e}}$ siècles) ${ }^{1}$, les réformes de Pierre le Grand dans l'armée russe, le développement de l'industrie minière et métallurgique ont favorisé l'arrivée de mots allemands. Au XIX 
siècle, au moment du boom industriel, le vocabulaire politique et social russe s'est aussi enrichi d'emprunts ou calques allemands, comme rabotadateL' (Arbeitgeber - employeur), rynok truda (Arbeitsmarkt - marché du travail) ${ }^{2}$. Malgré les guerres et les conflits, le XX ${ }^{\mathrm{e}}$ siècle a également contribué à cette forme d'enrichissement de la langue russe. D'après Roger Comtet ${ }^{3}$, les bouleversements politiques et sociaux ont entraîné des mutations dans le russe, notamment avec la révolution de 1917. Pour ce faire, Comtet s'appuie sur les recherches d'André Mazon qui a démontré la nette prédominance des emprunts allemands dans le langage des militants ouvriers, par l'intermédiaire du marxisme. Toujours cité par Comtet, Roman Jakobson a également relevé la tolérance par les Russes des emprunts allemands pendant la guerre et l'importance de leur impact dans le lexique scientifique. Nous verrons que les emprunts datant des siècles passés sont nombreux dans la presse actuelle.

3 Notre corpus est constitué d'articles issus de la presse généraliste, destinée à un public large et qui se compose, selon Valéry Kossov ${ }^{4}$, de trois catégories: les journaux strictement officiels dépendant du pouvoir, les journaux ouvertement opposés au régime et ceux qui tout en étant sous la pression du régime donnent une analyse alternative. Les exemples qui sont traités ici proviennent surtout des quotidiens Moskovskij komsomolec, Izvestija ou de l'hebdomadaire Profil ainsi que d'articles de presse cités dans le NacionaL'nyj korpus russkogo jazyka (Corpus national de la langue russe) ${ }^{5}$, incluant toute la presse à partir des années 1990.

Les journaux dits généralistes contiennent aussi des articles spécialisés destinés à des lecteurs intéressés par des sujets précis, par exemple économiques ou politiques. Dans ce cas, les journalistes créent un lexique codé destiné à des initiés, qui peuvent, par l'usage de ce code, se distinguer dans la société. Il s'avère donc que l'introduction de l'emprunt n'est pas toujours dictée par la nécessité mais aussi par la volonté de ces journalistes.

Pourquoi l'emprunt lexical?

Dans le présent article, nous nous appuierons sur la définition d'emprunt en tant que « forme d'expression qu'une communauté linguistique reçoit d'une autre communauté »", proposée par Vittore Pisani et reprise par Louis Deroy. Sachant que «forme d'expression" peut désigner un mot, mais aussi un tour syntaxique, un trait morphologique ou bien un son, il nous semble indispensable de préciser que nous étudierons ici les mots ou les expressions comprenant ces mots. Le verbe "recevoir» interpelle: quand et comment une communauté linguistique, ici la communauté linguistique russe, reçoit-elle tel ou tel mot allemand, par quels intermédiaires est-il introduit? Nous ne nous attarderons pas sur ces intermédiaires, mais examinerons plus finement l'intégration d'emprunts. Plusieurs critères entrent en jeu lorsqu'il s'agit de cette dernière dans la langue, phonologiques, morpho-syntaxiques et sémantiques. Dans notre étude, il sera question de la manière dont les emprunts allemands ont été adaptés aux niveaux phonétique, orthographique et morphologique par la langue russe. Or, nous accentuerons notre attention en particulier sur les critères sémantiques qui, selon Louis

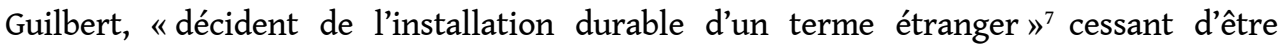
néologisme. L'emprunt, par suite de divers emplois, peut acquérir un sens supplémentaire et, par conséquent, une plus grande polysémie, différente de celle de la langue source.

7 De plus, parfois deux, voire plusieurs termes, quelle que soit leur origine, renvoyant au même objet ou phénomène cohabitent dans la langue russe. Pour illustrer cette 
cohabitation ainsi que l'extension de sens ou le glissement connotatif, nous étudierons quelques emprunts appartenant au lexique général et spécialisé : économique, politique ou encore argotique, ainsi que leurs emplois dans la presse actuelle.

\section{Les emprunts dans le lexique économique}

8 La terminologie économique figure parmi les lexiques de la langue russe qui ont été les plus imprégnés par l'allemand. Dès les XII ${ }^{\mathrm{e}}$-XIII ${ }^{\mathrm{e}}$ siècles, les échanges commerciaux entre l'Allemagne et la Rus' de Kiev ont largement contribué à l'apparition d'emprunts lexicaux. À la fin du XVII et au début du XVIII ${ }^{e}$ siècle, le développement du domaine financier a également été propice aux emprunts pour désigner de nouvelles réalités comme bank ( Bank - banque), birža (Börse - bourse), makler (Makler - courtier, agent de change), etc. Au $\mathrm{XX}^{\mathrm{e}}$ et au début du $\mathrm{XXI}^{\mathrm{e}}$ siècle, depuis la chute du régime communiste et suite à l'introduction de l'économie de marché, le lexique russe a continué à s'enrichir par des emprunts à l'allemand, ce qui a été démontré dans les récentes recherches des linguistes russes tels que Tokareva ou encore Svetlana Kepeščuk et AjguL' Timirgaleeva ${ }^{8}$.

Notre attention a été attirée par les emprunts allemands ayant subi une extension de sens en russe. Prenons l'exemple du lexème gešeft (Geschäft - affaire, commerce), emprunté par la langue russe à l'allemand à la fin du XIX siècle. Le Deutsches Wörterbuch (Dictionnaire allemand) de Gerhard Wahrig définit le mot Geschäft de la façon suivante :

Activité, occupation, travail (avec un but précis); métier, profession; résultat, activité lucrative, commerce, affaires, vente ; tâche, mission ; lieu de vente, sociétés commerciales, maison de commerce, magasin; (figuré - familier) satisfaire les besoins naturels. ${ }^{9}$

Le Deutsches Universalwörterbuch (Dictionnaire universel de la langue allemande) propose également plusieurs définitions du mot Geschäft :

1. a) Entreprise orientée vers le profit, transaction commerciale, commerce...,

b) Transactions commerciales, vente, chiffre d'affaires...,

c) Bénéfice (d'une entreprise commerciale), profit...

2. a) Société

b) Pièces, locaux, magasin...

3. Tâche, affaire... ${ }^{10}$

10 Observons maintenant le sens de gešeft relevé dans un article de la presse russe actuelle :

PervonačaL'no v osnove podobnoj linii ležalo stremlenie sdelaŤ « zakonnyj gešeft ».

Initialement, dans le fondement d'une ligne pareille, il y avait l'ambition de mener " une affaire légale "..."

L'ironie que l'auteur exprime ici envers cette affaire commerciale est soulignée par les guillemets. En effet, dans son Slovar' inostrannykh slov, vošedšikh $v$ sostav russkogo jazyka ( Dictionnaire des mots étrangers entrés dans la langue russe), Aleksandr Čudinov définit ce mot de la façon suivante :

(allemand) Se dit dans un sens ironique d'une affaire entreprise en vue d'un profit,

d'une spéculation de qualité inférieure reposant sur un mensonge. ${ }^{12}$

Aleksandr Babkin et Valentin Šendecov citent ce mot à côté d'un autre mot allemand Schwindel(mensonge, escroquerie)dans leur Slovar' inojazyčnykh vyraženij $i$ slov, upotrebljajuščikhsja $v$ russkom jazyke bez perevoda (Dictionnaire des expressions et des mots étrangers, employés dans la langue russe sans traduction) :

...Partout on escroque, on ment et on plume les autres. ${ }^{13}$ 
D'après la source citée par Babkin et Šendecov, on peut conclure que cet emprunt n'est pas nouveau car cette source remonte à $1913^{14}$. Le Nacionainyj korpus russkogo jazyka contient également ce mot dans des sources datant de 1880-1890 :

Moj otec priezžal sjuda delař gešeft na liniju, kogda stroilas' doroga...Gešeft ne pošel...Togda moj otec khodil v gorod s muzikoj et velel mne tože igraŤs soboj...

Mon père arriva ici pour faire des affaires sur la ligne où l'on construisait le chemin de fer. Ses affaires ne furent pas un succès. Alors mon père s'en alla à la ville pour faire de la musique et me demanda de l'accompagner... ${ }^{15}$

De plus, on emprunte non seulement un mot, mais aussi sa combinatoire. L'expression delař gešeft est calquée sur l'allemand mit etw ein gutes / schlechtes Geschäft machen (faire une bonne / mauvaise affaire). Il faut signaler que dans la presse ce mot ne s'emploie pas seulement dans le sens d'affaire commerciale, mais aussi dans celui de profit.

Kak možno dogadaŤsja, eti-to den'gi i eš̌ vernaja garantija pobedy MDM na aukcione pri privatizacii gospaketa (vpročem, daže esli privatizacija i otložitsja na neopredelennyj srok, ničto ne mešaet MeL'ničenko s Popovym izvlekaŤ svoj « gešeft » iz nynešnego polozenija - faktičeskogo vladenija gospaketom).

Comme on peut le deviner, cet argent est la garantie fidèle de la victoire du $\mathrm{MDM}^{16}$ aux enchères pour la privatisation des actions étatiques (d'ailleurs, même si la privatisation est repoussée à un délai inconnu, rien n'empêche MeL'ničenko et Popov de tirer «profit» de la situation actuelle - de la possession effective d'actions étatiques). ${ }^{17}$

11 En effet, dans le Bolšoj tolkovyj slovar' russkogo jazyka(Grand dictionnaire de la langue russe) de Kuznecov, cet emprunt est défini comme : «(langue parlée)Intérêt, profit, gain. Quel profit tirera-t-il de cela? $»^{18}$ Ce sens apparaît dans le discours des habitants d'Odessa pour lesquels il ne s'agit pas d'escroquerie, mais du profit tiré d'une affaire tout à fait légale : "Profit, bénef» (familier en yiddish). Il existe de nombreuses opinions sur le statut linguistique de ce qu'on appelle la "langue d'Odessa", basée sur le yiddish, le russe et l'ukrainien, considérée par les uns comme un dialecte et par les autres comme une langue indépendante. Inna Kabanen a consacré tout un travail de recherche à la " langue d'Odessa $»^{19}$, qui a été depuis sa fondation en 1794 par Catherine II une ville multiethnique, rassemblant de nombreux peuples : Grecs, Ukrainiens, Russes, Biélorusses, Moldaves, Italiens, Juifs, Polonais et Allemands. D'après Kabanen, qui cite Anna Veršik ou Nina Mečkovskaja, il s'agit d'une variante de la langue russe des Juifs (appelé également Jewish Russian) qui est finalement plus proche du yiddish par sa structure grammaticale que du russe ${ }^{20}$. Kabanen préfère, quant à elle, l'appeler «langue mixte », terme proposé par Peter Bakker pour nommer cette langue, formée sous l'influence de plusieurs ethnies. C'est notamment cette langue qui est devenue une source importante pour l'argot de la pègre en Russie. Odessa, ville portuaire et cosmopolite, accueillait bon nombre de commerçants malhonnêtes et toute sorte de contrebande. Dans ses Odesskie rasskazy (Récits d'Odessa) ${ }^{21}$, l'écrivain et dramaturge russe originaire de cette ville Isaak Babel' décrit avec fascination la vie des bandits à la fin du XIX et au début du XXe siècle. Comme le dit Daniel Aarao Reis dans son ouvrage Modernités nationales, modernités importées : Entre Ancien et Nouveau Monde (XIX ${ }^{e}-\mathrm{XXI}{ }^{e}$ siècles), le crime est devenu une banalité à Odessa, « dans cette ville, à certains moments, c'est comme si tous étaient des hors-la-loi. Mieux encore, comme si la loi n'existait pas... Se banalise ainsi l'arbitraire de nos bandits et la violence dont ils font usage $»^{22}$.

12 Aujourd'hui, les entrepreneurs n'hésitent pas à nommer leurs entreprises par le mot gešeft, qu'il s'agisse d'agences immobilières ou publicitaires, de sociétés de travaux publics ou même de banques: GEŠEFT, Finans-Gešeft, etc. Les textes publicitaires utilisent 
également ce mot pour inciter les entrepreneurs à ouvrir leur propre entreprise : «Sdelaj svoj gešeft! » ${ }^{23}$ (Crée ta propre entreprise !). Ces slogans publicitaires visent avant tout les Russes connaissant l'allemand qui comptent partir en Allemagne. Néanmoins, dans la presse actuelle, à l'exception des textes destinés aux locuteurs ciblés et initiés, gě̌eft perd son sens neutre de delo (affaire) et acquiert la connotation négative d'affaire louche. Il est nécessaire de comparer cet emprunt à celui fait au français : afera - arnaque, datant, selon le dictionnaire de Pavel Černykh, de la première moitié du XIX ${ }^{e}$ siècle ${ }^{24}$. Ce mot, également mentionné dans le Tolkovyj slovar' russkogo jazyka (Dictionnaire de la langue russe), est défini comme: "une entreprise, une affaire ou une action déloyale, frauduleuse $»^{25}$. En allemand, le mot Affäre a pour signification " une affaire désagréable, un incident embarrassant ou scandaleux $~^{26}$ ou encore "un litige, une liaison, une aventure amoureuse $»^{27}$. Même s'il ne s'agit pas d'une arnaque, ce mot est doté d'une valeur négative. Pourquoi alors le mot gešeft a-t-il été affecté d'une connotation péjorative, alors que l'emprunt afera évoquait déjà l'idée d'une affaire louche? Comme nous supposons qu'au départ gešeft ne possédait pas cette connotation péjorative, puisqu'il est toujours utilisé dans son sens direct d'affaire ou de profit, il ne s'agit pas d'un double emprunt. Le glissement connotatif a eu lieu plus tard. Aujourd'hui, la presse russe l'utilise le plus souvent dans sa connotation péjorative, comme le font, par exemple, le quotidien Moskovskij komsomolec ou l'hebdomadaire Profil, dans lesquels le mot gešeft est préféré au mot afera. Le mot gešeft, moins fréquent, semble mieux capter l'attention du lecteur par son exotisme. En utilisant des mots dotés d'une valeur négative dans le langage courant, les journalistes cherchent à attirer l'attention sur les imperfections de la situation politique et sociale, sans exprimer ouvertement leur opinion critique ni prendre parti dans leurs appréciations.

\section{Les emprunts dans l'argot russe}

13 Comme nous l'avons observé plus haut, l'argot russe contient plusieurs emprunts allemands. Le mot russe avtoritet (autorité) vient d'Autorität qui a les sens suivants en allemand:

1. Influence d'une personne ou d'une institution reposant sur la performance ou la tradition et la considération qui en découle.

2. Personnalité qui a une influence notable et qui fait autorité. ${ }^{28}$

D'après l'Etimologičeskij slovar' russkogo jazyka (Dictionnaire étymologique de la langue russe) de Max Fasmer, le mot avtoritetest utilisé pour la première fois en 1718, provenant du mot allemand Autorität, ce dernier ayant pour origine le mot latin auctoritas ${ }^{29}$. Dans le Novyj slovar' inostrannykh slov (Nouveau dictionnaire des mots étrangers), nous trouvons plusieurs sens du mot avtoritet :

Autorité, de l'allemand Autorität, du latin Auctoritas, pouvoir, influence. 1) importance universellement reconnue, influence; 2) celui dont l'importance est universellement reconnue, qui a une influence, par exemple il a une grande compétence (a de l'autorité) en médecine ; autorité dans le milieu criminel - chef d'une organisation criminelle, d'un grand groupe criminel. ${ }^{30}$

15 Nous constatons que le mot avtoriteta pris un sens supplémentaire : il a commencé à être employé dans l'argot du milieu pour désigner la personne à la tête d'une organisation criminelle et il apparaît, par conséquent, avec ce sens négatif dans la presse russe : «V Tveri ubit kriminaLnyj avtoritet Ibragim Azizov po kličke Arkaša $»^{31}$ (Le caïd Ibrahim, dont le surnom est Arkaša, a été tué à Tver). Même si l'adjectif « criminel » était absent, le 
lecteur pourrait comprendre qu'il s'agit d'un chef du milieu criminel grâce à l'emploi de termes tels que « tué » ou « surnom ».

Le mot abzac (Absatz), autre emprunt à l'allemand, s'est doté de plusieurs sens dans l'argot russe. Adopté par la langue russe dans le sens d'alinéa, ce lexème intègre la langue parlée où il gagne de nouveaux sens à la connotation tantôt positive, tantôt très négative. Voici quelques exemples tirés du Bolšojslovar' russkogožargona(Grand dictionnaire de l'argot russe) ${ }^{32}$ :

Čto ty nacepila? Kakoj-to abzac. Mitrofanov, Nikitina, MK.

Qu'est-ce que tu as mis là ? C'est une horreur ! Mitrofanov, Nikitina, MK.

Polnyj abzac. Slovar', paren', ty kupil - polnyj abzac!

Ce dictionnaire que tu as acheté, mon gars, il est vraiment super !

Aujourd'hui, dans la langue parlée, le mot Abzac se retrouve plus fréquemment chargé d'une connotation négative signifiant « une fin »:

Drugimi slovami, atomnyj abzac dlja Evropy možet nastupiŤ každuju minutu..$^{33}$

Autrement dit, l'apocalypse nucléaire pour l'Europe peut arriver d'une minute à

l'autre.

17 Nous observons la même évolution sémantique avec le mot finiš (fin), emprunté à l'anglais pour désigner dans le lexique sportif 1) La partie finale d'une compétition sportive ; 2) l'arrivée, la fin d'une épreuve ${ }^{34}$. Ce mot est ensuite utilisé dans la langue parlée et même dans les œuvres littéraires avec une connotation négative, évoquant quelque chose d'inéluctable :

Posle etogo i načalos', brat načal piř...v obščem, eto yže byl finiš..$^{35}$

Après cela, tout a commencé, mon frère s'est mis à boire et c'était là le début de la

fin.

18 Le lexique technique allemand a fourni à la langue russe plusieurs termes comme šajba ( Scheibe - palet), šakhta (mine), šifer (ardoise), cekh (atelier) ${ }^{36}$. Si nous analysons le mot šajba , emprunté à l'allemand pour nommer une pièce en forme de disque ayant une fonction technique, nous nous apercevons qu'il a acquis, par extension, le sens de palet dans le hockey sur glace. Dans la langue allemande, le mot Scheibe a, entre autres, le sens de cible dans le lexique sportif ou militaire. En revanche, en russe, ce sens serait exprimé par le mot mišen'.Dans le langage parlé, on utilise le mot šajbapour encourager les joueurs de hockey à marquer un but.

19 De plus, dans l'argot du milieu ce mot désigne plusieurs choses: "Une place ronde, une pièce de monnaie, l'argent, l'acte sexuel $»^{37}$. Ces sens seraient peut-être venus, par analogie, du sens suivant du mot Scheibe: "(Dans le domaine technique) pour une fonction technique spécifique, disque (par exemple, disque de frein, rondelle de joint) généralement circulaire [avec un trou prévu en son centre] et destiné à être incorporé en tant que pièce rotative dans une machine ou autre. $1^{38}$

20 Par ailleurs, les mots šakhta, šifer ou cekh se sont également dotés de nouveaux sens par leur utilisation dans l'argot du milieu et ont de ce fait souvent acquis une valeur péjorative. Les emprunts lexicaux comme gešeft, avtoritet, abzac ou šajba, qui ont vu leur sens s'étendre à des significations différentes de celles de la langue source, illustrent le phénomène de l'emprunt interne qui mène à une plus grande polysémie.

21 L'argot russe a maintes fois eu recours aux langues étrangères, comme le polonais, l'allemand ${ }^{39}$ ou le français ${ }^{40}$. Les emprunts, ne possédant souvent aucune connotation dans leurs lexiques d'origine, acquièrent un sens nouveau dans la langue russe comme blat, šprekhař, šprekhanuŤ (sprechen), abkhaL'ten (abhalten), etc. Analysons le mot blat (initié, 
complice) qui, selon le dictionnaire de Fasmer ${ }^{41}$, est d'origine judéo-allemande. Dans l'allemand courant, le mot Blatt signifie la feuille d'un végétal et, par extension, la feuille de papier, un journal ou un jeu de cartes ${ }^{42}$. Lors de son passage de l'allemand au russe, blat a perdu ses sens dès le début, contrairement au mot avtoritet,et a été fixé dans le Tolkovyj slovar' russkogo jazyka avec les sens suivants :

1. Argot des voleurs.

2. Connaissances, relations qu'on peut utiliser à des fins personnelles ou pour en tirer profit (langage populaire). ${ }^{43}$

Ce mot a été fixé pour la première fois dans le dictionnaire de Vasilij Trakhtenberg Blatnaja muzyka : Žargon tjur'my. Po materialam, sobrannym $v$ peresyL'nyx tjur'max (Argot des voleurs et de prison : selon les documents recueillis dans les prisons de transit) en $1908^{44}$ :

Tout crime, de n'importe quel type: vol,meurtre, escroquerie, cambriolage, contrebande, distillation clandestine, fabrication et commercialisation de fausse monnaie, etc. - possède en argot un seul nom générique : «blat »

Le sens du mot blat compris comme des connaissances ou des relations s'est répandu pendant la période soviétique dans les années 1930. À cette époque, la pénurie permanente entraîne le phénomène de faveur, et connaître des vendeurs ou des apparatchiks permet aux gens d'obtenir des marchandises et des services plus rapidement ou encore ce qui est inaccessible. Ce sens serait en lien avec le mot Blatt, feuille de papier, comme code, signe d'appartenance à un groupe d'initiés. Vasilij Trakhtenberg souligne également que le mot blat désigne, entre autres, une affaire illégale, comme une contrebande.

Si, dans ce deuxième sens, le substantif blat et son dérivé continuent à être employés dans la presse russe actuelle, dans le premier sens, c'est l'adjectif blatnoj qui est plus utilisé :

Tak čto $\mathrm{v}$ sukhom ostatke polučaetsja, čto EGE ne tol'ko ne otmenil blat, no i uzakonil ego. ${ }^{45} \mathrm{Ainsi}$, le résultat c'est que l'examen d'État unifié non seulement ne supprime pas le piston, mais va jusqu'à le légitimer.

Tjur'ma davno vošla v naš byt, našu kuL'turusvoej blatnoj čaš̌ju. ${ }^{46} \mathrm{La}$ prison est entrée depuis longtemps dans notre quotidien et dans notre culture par le biais de l'argot des voleurs.

Pour donner un autre exemple, citons le couple de mots ayant la même racine šprekhă̌ / šprekhanuŤ. Ces mots viennent du verbe allemand sprechen, tout à fait neutre, privé d'expressivité. En russe, šprekha Ť s'utilise dans le langage parlé pour signifier " parler une langue étrangère ${ }^{47}$ et šprekhanư̌, dans l'argot du milieu, pour "dénoncer ». Comme le démontre le slaviste allemand Tilman Bergerdans dans son étude "Sprechtit, šprekhaŤund andere lustige verba dicendi $»^{48}$ (Sprechtit, šprekhaŤ et autre drôle de façon de dire "dire»), ces mots appartiennent à la «langue Quelia», formée par des russophones juifs habitant en Allemagne, en Autriche et en Suisse. Ils ont effectivement créé leur propre langue pour communiquer en se fondant sur la grammaire russe et le lexique allemand.

\section{Les emprunts dans le lexique politique et social}

Le lexique politique et social russe a également emprunté plusieurs termes à l'allemand, parmi lesquels nombreux sont d'origine latine : agent (Agent, du latin agentis), agentura ( Agentur), lozung (Losung), ljumpen (Lumpenproletariat), nejtralitet (Neutralität, du latin neuter ), paritet (Parität, du latin paritas), putč (Putsch), bundestag (Bundestag - Parlement), bundesver (Bundeswehr - armée), bundesrat (Bundesrat - chambre du Parlement), kancler ( 
Kanzler - chancelier), fjurer (Führer), policaj (Polizei - police) et d'autres. Le linguiste russe Leonid Krysin souligne que le mot policaj (Polizei), après avoir glissé dans le russe pendant la Seconde Guerre mondiale, a acquis une connotation négative en désignant un " policier, collaborateur de la police, nommé par le commandement allemand sur le territoire occupé $»^{49}$. Ainsi, on assiste au glissement connotatif du mot Polizei qui ne possède aucune connotation péjorative en allemand. Il est intéressant de noter qu'aujourd'hui ce mot s'emploie toujours dans la presse russe, tout en gardant une valeur péjorative, pour parler de la police russe en général :

« Perenesli sud - policaj ne javilsja » ${ }^{50}$

On a ajourné le délai du jugement au tribunal - le policier n'est pas venu.

26 Il faudrait également se pencher davantage sur l'emprunt à l'allemand des mots agent et agentura. D'après l'Etimologičeskijslovar' russkogojazyka (Dictionnaire étymologiquede la langue russe)de Galina Cyganenko, le mot agent, apparu dans la langue russe au début $\mathrm{XVII}^{\mathrm{e}}$ siècle, provient de l'allemand Agent avec le sens de "plénipotentiaire ${ }^{51}$, qui est défini dans le Duden online de la façon suivante :

1. Personne missionnée secrètement par un gouvernement, une organisation militaire ou autres, devant exécuter des missions définies, la plupart du temps illégales; espion.

2. (Écon. Vieilli) a) Personne qui fait et conclut des affaires, la plupart du temps sur la base de provisions ; représentant de commerce. b) Personne dont le métier est de rechercher des engagements aux artistes. ${ }^{52}$

L'actuelTolkovyj slovar' russkogo jazyka mentionne trois définitions de l'emprunt agent similaires à celles d'Agent en allemand :

1. Personne, chargée par un établissement de régler des tâches et des missions de service.

2. Personne qui agit dans les intérêts de quelqu'un.

3. Voirespion. ${ }^{53}$

En revanche, le mot Agentur, qui a donné l'emprunt agentura au russe, ne signifie en allemand que :

1. (particulièrement en économie) Institution qui représente quelqu'un, quelque chose, qui procure quelque chose.

2. Forme abrégée pour agence de presse..$^{54}$

En russe, le mot agentura désigne le service des renseignements (razvedyvateinaja ou sysknaja služba), qui est nommé en allemand die Agenten, comme le précise Karl Gottlieb dans son Wörterbuch der "Falschen Freunde des Übersetzers" (russisch-deutsch/ deutschrussisch) (Dictionnaire des faux amis du traducteur [russe-allemand / allemand-russe]) $)^{55}$. Dans les sources russes, on situe l'emploi de ce mot au début du XXe siècle et il est toujours utilisé notamment dans la presse actuelle :

V bor'be s narkotikami ro' agentury črezvyčajna vysoka, odnako, kak my uze govorili, eto agentura sovsem drugogo roda. ${ }^{56} \mathrm{Dans}$ la lutte contre les drogues, le rôle des agents est extrêmement important, cependant, comme nous l'avons déjà dit, il s'agit d'agents d'un tout autre type.

\section{L'adaptation phonétique, orthographique et morphosyntaxique}

Lors de l'adaptation phonétique des emprunts qui se traduit par une adaptation orthographique émerge le problème de la graphie, comme le souligne Christine 
Bracquenier dans son article "L'adaptation des emprunts lexicaux du français par la langue russe : de Karamzin à Akunin ${ }^{57}$. Nous observons le même phénomène pour les emprunts à l'allemand. Dans les exemples précédemment cités, comme pour le mot gešeft, nous voyons l'évolution de l'adaptation vers la translittération ou la transcription, qui remplace l'utilisation des mots originaux. En effet, pour une meilleure assimilation des emprunts, la transcription est beaucoup plus commode, tandis que l'utilisation des mots originaux est réservée aux énonciateurs souhaitant démontrer leur appartenance à un groupe ou à une classe sur l'échelle sociale ou encore concerne les noms propres des marques des vêtements, des produits de beauté, de l'électroménager, des noms des sociétés, etc., comme «Braun », "Nivea », Schwarzkopf», "Deutsche Bank». Ces noms sont également transcrits en cyrillique et nous retrouvons l'emploi des deux orthographes dans la presse russe actuelle. D'après Timirgaleeva ${ }^{58}$, dans les journaux spécialisés ou la publicité, les noms sont conservés en lettres latines, tandis que les œuvres littéraires et les dictionnaires préfèrent les transcriptions en cyrillique. Par ailleurs, nous avons rencontré le cas de la transcription et de l'orignal dans le même article de presse :

Pavel Teplukhin s 1 oktjabrja stanovitsja glavnym ispolniteL’nym direktorom Dojče Banka v Rossii, ob'javil v sredu bank. On budet podčinjatsja Štefanu Ljajtneru, členu pravlenija i členu ispolniteL'nogo komitetaa gruppy, glave podrazdelenij Deutsche Bank AG. ${ }^{59}$

À partir du $1^{\mathrm{er}}$ octobre, Pavel Teplukhin devient le principal directeur exécutif de la banque allemande "Deutsche Bank» en Russie, a annoncé la banque mercredi. Il rendra compte à Stefan Leithner, membre du Conseil d'administration et du comité exécutif du groupe, chef des succursales de Deutsche Bank AG.

Concernant l'adaptation morphologique, le russe emprunte beaucoup de substantifs à partir desquels il peut ensuite former des dérivés, selon les règles de formation de mots en russe, par exemple blat / blatnoj. Les verbes allemands sont également transformés en verbes russes, souvent à l'aide du suffixe -ova ce qui n'est pas le cas des verbes šprekhař / šprekhanuॅ̌, formés avec la base špreha, avec ou sans le suffixe -nu Et la désinence - ̌r. Cette possibilité qu'ont les emprunts allemands de servir de base à une dérivation indique le haut degré de leur intégration dans la langue russe.

31 Le choix du genre n'est pas toujours déterminé par l'article du mot allemand, comme pour das Geschäft ou die Autorität. Ces mots, neutre ou féminin à l'origine, prennent le genre masculin en russe, puisqu'ils se terminent par une consonne.

\section{Conclusion}

D'après cette brève étude, nous pouvons constater que la presse russe a puisé beaucoup d'emprunts lexicaux à l'allemand. Ils ont joué un rôle non négligeable dans la formation du lexique russe, parce qu'ils se sont dotés le plus souvent d'un nouveau sens, comme dans le cas de šajba ou avtoritet. On constate également un large éventail d'emprunts comme blat ou agentura qui, par extension ou glissement connotatif, ont intégré la langue russe avec des sens différents de ceux de l'original. La traduction de ces derniers présente un problème particulier, lié aux « faux amis ». Afin de le contourner, le traducteur se doit de porter son attention sur les contextes et sur les domaines d'utilisation.

La fréquence des connotations péjoratives ou ironiques caractérise la plupart du temps les emprunts étudiés, retrouvés dans les articles de presse généralistes. Les journalistes 
cherchent ainsi à présenter leur image des événements politiques et sociaux actuels en s'adressant au lecteur initié. L'emploi courant des emprunts à valeur négative dans la presse est étroitement lié à leur utilisation dans le langage de la population russe dans la vie de tous les jours. Ironique ou hostile, elle reflète la tendance de l'opinion publique, sans pour autant proposer de solutions aux problèmes de la société.

C'est souvent l'adoption du terme dans le langage argotique qui crée ces connotations car son lexique très expressif est marqué par l'attribution de valeurs positives ou négatives. Comme nous avons pu l'observer, ce phénomène concerne non seulement les emprunts à l'allemand, mais aussi les emprunts à l'anglais ou au français, et si certains restent à la mode et sont toujours d'usage fréquent, d'autres sont délaissés, le plus souvent remplacés par de nouvelles créations Dans des travaux de recherches ultérieurs, il conviendrait d'étudier l'interaction entre les emprunts à l'anglais, à l'allemand et au français, ainsi que l'évolution de leur emploi.

\section{NOTES}

1. Tokareva I., Adaptacija nemeckikh leksičeskikh zaimstvovanij $v$ russkom literaturnom jazyke: $\mathrm{Na}$ materiale istočnikov rubeža XIX - XX vv. (L'adaptation des emprunts lexicaux dans la langue russe littéraire: Sources des $\mathrm{XIX}^{\mathrm{e}}$ et $\mathrm{XX}^{\mathrm{e}}$ siècles), thèse de doctorat, Tjumenski gosudarstvennyj universitet, Tjumen', 2003, $175 \mathrm{p}$.

2. Ibid., p. 47.

3. Comtet R., Les emprunts linguistiques dans le débat sur la langue en Russie : d'une révolution à l'autre, in : Slavica occitania, 1996/3, pp. 11-35.

4. Kossov V., «La langue russe à l'épreuve de la crise économique de 2007-2009: les apports linguistiques au travers de la presse », ILCEA [En ligne], 15 | 2012, mis en ligne le 30 janvier 2012, consulté le 16 novembre 2013. URL : http://ilcea.revues.org/1195.

5. NacionaL'nyj korpus russkogo jazyka (Corpus national de la langue russe), URL: http:// www.ruscorpora.ru.

6. Deroy L., L'emprunt linguistique, Les Belles Lettres, Paris, 1956, p. 18.

7. Guilbert L., La créativité lexicale, Librairie Larousse, Paris, 1965, p. 98.

8. Kepeščuk Sv., Nemeckie leksičeskie zaimstvovanija kak istočnik popolnenija russkogo leksikona (Les emprunts lexicaux, source pour le lexique russe), Tjumenski gosudarstvennyj universitet, Tjumen', 2001, 199 p.; Tokareva I., op.cit.; Timirgaleeva A., Inojazyčnaja leksika nemeckogo proiskhoždenija v russkom jazyke noveišego perioda (Le lexique étranger d'origine allemande dans la langue russe de la période actuelle), Kazanskij gosudarstvennyj tekhničeskij universitet im. A. N. Tupoleva, Kazan', 2006, 179 p.

9. Wahrig G., Deutsches Wörterbuch (Dictionnaire de la langue allemande), Bertelsmann LexikonVerlag, Gütersloch, 1968 et 1972, p. 1476. Toutes les traductions, de l'allemand comme du russe, sont de l'auteur de cet article.

10. Duden K., Deutsches Universalwörterbuch (Dictionnaire universel de la langue allemande), Dudenverlg, Mannheim-Vienne-Zürich, 1989, p. 595.

11. Rostovskij M., «Ukrožčenie Rossii : čego Amerika na samom dele khočet ot Kremlja. ČasŤ II» (La Russie apprivoisée: ce que les États-Unis veulent réellement du Kremlin), in : Moskovskij 
komsomolec, $\mathrm{n}^{\circ} 26$ 234, URL : http://www.mk.ru/politics/world/article/2013/05/20/856783ukroschenie-rossii-chego-amerika-na-samom-dele-hochet-ot-kremlya.html, consulté le 12 juin 2013.

12. Čudinov A. N., Slovar' inostrannykh slov, vošedšikh v sostav russkogo jazyka (Dictionnaire des mots étrangers, entrés dans la langue russe), V.I. Gubinskij, Saint-Pétersbourg, 1910, p. 157.

13. Babkin A. M. \& Šendecov V. V., Slovar' inojazyčnykh vyraženij $i$ slov, upotrebljajuščikhsja v russkom jazyke bez perevoda (Dictionnaire des expressions et des mots étrangers, employés dans la langue russe sans traduction), Kniga 2, « KVOTAM », Saint-Pétersbourg, 1994, p.558.

14. Il s'agit de la citation de Vladimir Spacovič, juriste, historien et critique littéraire russe d'origine polonaise, qui décrit son voyage en Dalmatie, Bosnie et Herzégovine en 1882.

15. Zlatovratskij N. N., «Siroty 305-j versty» (Les orphelins de la verste 305), URL : http:// www.ruscorpora.ru, consulté le 14 juin 2013.

16. La Banque russe Moskovskij delovoj mir (Monde des affaires de Moscou).

17. Gotova N., «Černo-buraja monopolija » (Le monopole de l'or noir), URL: http:// www.profil.ru, publié le 17 mars 2003, consulté le $1^{\text {er }}$ juin 2013.

18. Kuznecov S. A., Bolšoj tolkovyj slovar' russkogo jazyka (Grand dictionnaire de la langue), Norint, Saint-Pétersbourg, 1998, p. 201.

19. Kabanen I., Vvedenie $v$ osobennosti odesskogo jazyka (Introduction aux particularités de la langue d'Odessa), Kevat, 2008, 50 p., URL: http://www.helsinki.fi/venaja/opiskelu/graduja/ kabanen.pdf, consulté le 15 juin 2013.

20. Ibid., p.10.

21. Babel' I., Odesskie rasskazy (Récits d'Odessa), Leninizdat, Saint-Pétersbourg, 2012, 192 p. Ces textes datent de 1931.

22. Aarao Reis D. \& Rolland D., Modernités nationales, modernités importées : Entre Ancien et Nouveau Monde XIX-XXI siècle, L'Harmattan, Paris, p. 63

23. In : Homes-Collection.com, URL : http://homes-collection.com/2013/articles/business/sdelaysvoy-gesheft/, consulté le 12 juin 2013.

24. Černykh P., Istoriko-etimologičeskij slovar' russkogo jazyka (Dictionnaire historico-étymologique de la langue russe), t. 1, Russkij jazyk, Moscou, 1993, p. 59.

25. Ožegov S. I. \& Švedova N. Y., Tolkovyj slovar' russkogo jazyka (Dictionnaire de la langue russe), OOO izd. ELPIS, Moscou, 2003, p.32.

26. Duden online, URL: http://www.duden.de/rechtschreibung/Affaere, consulté le $1^{\text {er }}$ juin 2013.

27. Wahrig G., Deutsches Wörterbuch (Dictionnaire allemand), Bertelsmann Lexikon-Verlag, Gütersloch 1968 et 1972, p. 369.

28. Duden online, URL : http://www.duden.de/rechtschreibung/Autoritaet, consulté le 2 juin 2013.

29. Fasmer M., Etimologičeskij slovar' russkogo jazyka (Dictionnaire étymologique de la langue russe), Astre', AST, ACT, Moscou, 2004, 60 p.

30. Zakharenko E. N., Komarova L. N. \& Necaeva I. V., Novyj slovar' inostrannykh slov (Nouveau dictionnaire des mots étrangers), Azbukovnik, Moscou, 2008, p. 26.

31. Sokolov-Mitrič D., «SmerŤ za Kruga. V Tveri rasstreljan čelovek, kotoromu molva pripisyvaet ubijstvo

znamenitogo šanson'e » (A Tver l'homme qui a, selon la rumeur, tué le célèbre chanteur Krug, a été fusillé), in : Izvestija, 20 septembre 2002, URL : http://www.ruscorpora.ru, consulté le 10 juin 2013.

32. Mokienko V. M. \& Nikitina T. G., Bolšoj slovar' russkogo žargona (Grand dictionnaire de l'argot russe), Norint, Moscou, 2000, p. 29. 
33. "ČernobyL'skaja radosŤ Ukrainy " (La joie de Tchernobyl en Ukraine), in : KriminaL'naja khronika (Chronique criminelle), publié le 24 juillet 2003, URL : http//www.ruscorpora.ru, consulté le 12 juin 2013.

34. Ožegov S. I. \& Švedova N. Y., op. cit., p. 852.

35. Katerli N., «Dnevnik slomannoj kukly» (Le journal de la poupée cassée), in : Zvezda, 2001, URL : http://www.ruscorpora.ru, consulté le 14 juin 2013.

36. Timirgaleeva A., op. cit., p. 38

37. Mokienko V. M. \& Nikitina T. G., op. cit., p. 680.

38. Duden online, URL : http://www.duden.de/rechtschreibung/Scheibe, consulté le 15 juillet 2013.

39. Dans le dictionnaire de Vasilij Trakhtenberg Blatnaja muzyka: Žargon tjur'my. Po materialam, sobrannym $v$ peresyL'nyx tjur'max (Argot des voleurs et de prison : selon les documents recueillis dans les prisons de transit), on observe un grand nombre de mots ou d'expressions provenant de l'allemand : "cirlih ( en allemand zierlich - menu, fin), guten-morgen (Guten Morgen), štui'n (en allemand :stehlen - voler), frajer, fraer, freier... " in: Trakhtenberg V. F., Blatnaja muzyka, op. cit., sous la direction de I. A. Baudouin de Courtenay, tip. A. G. Rozen, Saint-Pétersbourg, 1908, 116 p.

40. T. M. Nikolaeva cite les emprunts au français dans l'argot russe du milieu : «Fors - den'gi (en français. force) dlja bleziru (en français plaisir) et autres. ». Nikolaeva T. M., « Boduen de Kurtene redaktor slovarja V.F. Trakhtenberga "Blatnaja muzyka, Žargon tjurmy" (Baudouin de Courtenay, rédacteur du dictionnaire de V. F. Trakhtenberg "Argot des voleurs et de prison", in: Russkaja $i$ sopostoviteL'naja filologija: LingvokuL'turologičeskij aspekt (Philologie russe et contrastive: aspect culturo-linguistique), izdanie Kazanskogo universiteta, Kazan', 2004, pp. 176-181.

41. Fasmer M., op. cit., p.172.

42. Duden online, URL: http://www.duden.de/suchen/dudenonline/Blatt, consulté le 15 juin 2013.

43. Ožegov S. I. \& Švedova N. Y., op. cit., p. 50.

44. Trakhtenberg V. F., op. cit., pp. 7-8.

45. AmeL'kina A., «PusŤ otdykhajut... na Solovkakh » (Qu'ils se reposent... aux îles Solovki), in : Izvestija, publié le 24 juin 2003, URL : http://www.ruscorpora.ru, consulté le 14 juin 2013.

46. Gilinskij Ja., "Prizonizacija po-rossijski » (L'influence de la prison sur la vie de la société russe), in : Otečestvennye zapiski (Annales de la patrie), 2003, http://www.ruscorpora.ru, consulté le 18 juin 2013.

47. Khimik V. V., BoL'šoj slovar' russkoj razgovornoj ekspressivnoj leksiki (Grand dictionnaire du lexique expressif de la langue parlée), Norint, Saint-Pétersbourg, 2004, p. 741.

48. Berger T., «Sprechtit, šprekhaר̌ und andere lustige verba dicendi » (Sprechtit, šprekhaŤ et autre drôle de façon de dire «dire»), URL : http://homepages.uni-tuebingen.de/tilman.berger/ Handouts/Salzburg2009.pdf, consulté le 16 juin 2013.

49. Krysin L., Inojazyčnye slova v sovremennom russkom jazyke (Les mots étrangers dans la langue russe d'aujourd'hui), Nauka, Moscou, 1968, p. 132.

50. http://lenta.ru/video/2013/04/14/kosenko1/,publié le 14 avril 2013, consulté le 13 juin 2013. 51. Cyganenko G.P., Etimologičeskij slovar' russkogo jazyka (Dictionnaire éthymologique de la langue russe), Radjans'ka škola, Kiev, 1989, p. 13.

52. Duden online, URL : http://www.duden.de/rechtschreibung/Agent, consulté le 8 juin 2013.

53. Ožegov S. I. \& Švedova N. Y., op. cit., p. 21.

54. Duden online, URL : http://www.duden.de/rechtschreibung/Agentur, consulté le 8 juin 2013.

55. Gottlieb K. G. M., Wörterbuch der «Falschen Freunde des Übersetzers» (russisch-deutsch / deutschrussisch) (Dictionnaire des faux amis du traducteur, russe-allemand, allemand-russe), Russkij jazyk, Moscou, 1985, p. 37. 
56. Nikitinskij L., «Proščaj, policejskij...» (Adieu, le policier) in : Moskovskie novosti, 2003, URL : http://www.ruscorpora.ru, consulté le 18 juin 2013.

57. Bracquenier Chr., "L'adaptation des emprunts lexicaux du français par la langue russe : de Karamzin à Akunin», in :Les emprunts lexicaux du français dans les langues européennes, Editura Universitaria, Craiova, 2011, pp. 1-9.

58. Timirgaleeva A., op. cit., p. 55.

59. Orlova Ju., «Teplukhin vozglavil Dojče Bank v Rossii » (Teplukhin prend la direction de la Deutsche banque en Russie), in: Gazeta.ru, publié le 26 septembre 2012, URL: http:// www.gazeta.ru/financial/2012/09/26/kz_4788625.shtml, consulté le 20 juin 2013.

\section{RÉSUMÉS}

Le présent article est consacré à l'analyse des emprunts allemands dans la presse russe actuelle. Cette étude consiste à démontrer l'évolution sémantique des mots allemands intégrés aux différents moments de l'histoire dans le lexique général et spécialisé russe: économique, politique ou encore argotique. Il sera donc question de l'extension de sens ou glissement connotatif des emprunts. On voit que ces derniers ne sont pas nouveaux dans la presse et que leur emploi a évolué avec le temps. On constate également que l'usage des emprunts à valeur négative est une tendance du monde journalistique.

This article proposes to analyse German loan words in current the Russian written press. This study consists of showing the semantic evolution of German words were integrated in at different historical moments in the Russian general or specialized lexicon: economical, political and slang. The article analyses the question of sense extension or connotative change of loan words. We can see that these words are not new in the press and their use changed with time. We note as well that the press has a tendency to use the loan words with a negative connotation.

\section{INDEX}

Index chronologique : XXe siècle, XXIe siècle

Index géographique : Russie

Mots-clés : emprunt, extension de sens, glissement connotatif, presse, sémantique

Keywords : connotative change, loan words, press, semantic, sense extension

\section{AUTEUR}

\section{VALENTINA CAILLAT-TUZHIKOVA}

Chargée d'enseignement, Université Stendhal-Grenoble 3, Docteur es Langues, Littératures et Civilisations, CERCLE EA 7342

valentina.caillat@u-grenoble3.fr 